\title{
iTRAQ-based comparative proteomics analysis reveals specific urinary biomarkers for various kidney diseases
}

Juan Jin

Department of nephrology, Zhejiang Provincial People's Hospital, Zhejiang Jianguang Gong

Department of nephrology, Zhejiang Provincial People's Hospital, Zhejiang

Li Zhao

Department of nephrology, Zhejiang Provincial People's Hospital, Zhejiang

Yiwen Li

Department of nephrology, Zhejiang Provincial People's Hospital, Zhejiang

Qiang He ( $\square$ qianghe1973@126.com )

Zhejiang Provincial People's Hospital https://orcid.org/0000-0003-3811-8226

Research article

Keywords: Kidney disease, Urinary proteomics, differentially expressed proteins, Biomarker discovery

Posted Date: August 31st, 2019

DOI: https://doi.org/10.21203/rs.2.13809/v1

License: (c) (i) This work is licensed under a Creative Commons Attribution 4.0 International License. Read Full License

Version of Record: A version of this preprint was published at Biomarkers in Medicine on July 1st, 2020. See the published version at https://doi.org/10.2217/bmm-2019-0556. 


\section{Abstract}

Background Urinary proteomics has been extensively applied to investigate renal diseases including acute kidney injury (AKI), chronic kidney disease (CKD), IgA nephropathy (IgAN) and diabetic CKD. However, differential urinary proteome studies have not been reported for multiple diseases. The present study was aimed to explore early clinical diagnosis biomarkers for patients with AKI, AKI+CKD, diabetic CKD, non-diabetic CKD with IgAN and non-diabetic CKD without IgAN. Methods Differentially expressed proteins (DEPs) were screened by iTRAQ labeling and 2-D LC-MS/MS. Bioinformatics analysis was performed by subsequent $\mathrm{GO}$ enrichment and KEGG pathway analysis. DEPs were authenticated by ELISA assay. Results $156,156,286,187$ and 184 differentially abundant proteins were identified in patients with $A K I, A K I+C K D$, diabetic CKD, and non-diabetic CKD with or without IgAN. Comparative analysis indicated that 34, 35 and 17 unique DEPs were found in AKI, AKI+CKD and CKD samples, respectively. 91 and 14 specific DEPs were screened out in diabetic CKD and non-diabetic CKD. In comparison with Non-diabetic CKD with IgAN (38 DEPs), 47 unique urinary proteins were found in Nondiabetic CKD without IgAN. Among these DEPs, urinary SAA1 and HGFAC were only unregulated in AKI and Non-diabetic CKD without IgAN implying that they might be employed as the potential indicators of the two diseases. C5, APOC1 and Reg3A upregulation was not exclusively expressed in each disease which suggested that they could not be used for biomarker to distinguish one disease from the other. Conclusion Collectively, this research contributes to the urinary biomarker discovery from multiple renal diseases.

\section{Background}

Kidney disease includes acute kidney injury (AKI) and chronic kidney disease (CKD), often resulting in renal failure, especially among hospitalised patients. Causes of kidney disease are numerous and complex, including deposition of immunoglobulin A $(\lg A)$ antibodies in the glomerulus, termed $\lg A$ nephropathy (1), diabetes mellitus leading to diabetic nephropathy (DN) (2), and high blood pressureinitiated hypertensive nephropathy (3). To date, 400 different rare renal diseases have been reported. Therefore, it is very important to identify reliable biomarkers that facilitate early diagnosis of kidney diseases and help distinguish one type of disease from another.

The urinary proteome consists of blood and renal-derived proteins that can reflect systemic and renal disorders. Urinary proteomics approaches have been widely used to differentiate disease types and stages, and to assist the discovery of diagnostic biomarkers, especially for kidney disease. AKI involves an abrupt loss of kidney function that develops within 7 days, and $~ 5-10 \%$ of AKI patients can progress into CKD, and even end-stage kidney disease (ESRD), depending on the cause $(4,5)$. Clinically, AKI is often diagnosed based on the elevation of blood urea nitrogen and creatinine, or the amount of urine per $24 \mathrm{~h}$ period. Recently, numerous novel AKI biomarkers, such as neutrophil gelatinase-associated lipocalin (NGAL) (6), kidney injury molecule 1 (KIM-1) (7) interleukin 18 (IL-18) (8), liver-type fatty acid-binding protein (L-FABP) (9) and activin A (10), have been identified in urine and found to contribute to early detection, differential diagnosis, and prognosis for AKI. 
CKD, as a common type of renal disease, is characterised by a gradual loss of kidney function over a period of months or years, and mainly includes DN, non-diabetic nephropathy (NDN) and other types of nephropathy. Reduction in estimated glomerular filtration rate (eGFR) is regarded as the best predictor of $C K D$, but this is only effective during the latter stages of renal disease (11). Cystatin $C$ and creatinine are protein biomarkers for CKD, and cystatin $C$ may improve eGFR in the progression of CKD (12). Evidence suggests that urinary 8 - hydroxyl -2' - deoxyguanosine (8-OHdG) (13), transferrin(14), type IV collagen (15), NGAL (16), and monocyte chemoattractant protein-1 (MCP-1) (17) can be used as biomarkers to predict patients with $\mathrm{DN}$.

IgA nephropathy (IgAN), characterised by deposition of IgA antibodies in the glomerulus, is the most common form of glomerulonephritis throughout the world (18). Based on nano-liquid chromatographytandem mass spectrometry (LC-MS/MS), 2D-electrophoresis-matrix-assisted laser desorption ionisationtime-of-flight/time - of-flight (2DE-MALDI-TOF-TOF) mass spectrometry (MS) and enzyme-linked immunosorbent assay (ELISA) analytical techniques, numerous studies have identified many noninvasive diagnostic markers in urinary excretions from relevant proteins in IgAN, including GP2, protocadherin (19), albumin fragments, LG3 (20) and others. Although a growing number of urine protein markers for kidney disease have been identified using advanced biotechnological methods, only a few urine protein biomarkers have been validated and used for clinical purposes. Additionally, case-crossover studies among multiple renal diseases to identify specific urinary protein markers that can accurately distinguish between different kidney diseases have not been reported.

Herein, based on isobaric tag for relative and absolute quantitation (iTRAQ) labelling and LC-MS/MS, we identified differentially expressed proteins (DEPs) in urine from patients with AKI, AKI+CKD, diabetic CKD, and non-diabetic CKD with or without IgAN. We subsequently performed Gene Ontology, GO term, and Kyoto Encyclopaedia of Genes and Genomes (KEGG) pathway analyses using the identified DEPs. Finally, we identified specific DEPs in each of the five types of kidney diseases. In summary, we identified several novel urinary protein biomarkers that can distinguish and assist classification of multiple kidney diseases.

\section{Methods}

\section{Subjects}

A total of 300 urine samples were obtained for urinary proteomic profiling from healthy volunteers $(n=$ $50)$ and patients with early AKI $(n=50)$, AKI with chronic kidney disease (AKI+CKD; $n=50)$, diabetic CKD $(n=50)$, non-diabetic CKD with IgAN $(n=50)$, and non-diabetic CKD without IgAN $(n=50)$. Inclusion criteria for early AKI (Stage I) were an increase in blood creatinine by $26.2 \mu \mathrm{mol} / \mathrm{l}$, or 1.5-1.9-fold in $48 \mathrm{~h}$ and urine volume less than $0.5 \mathrm{ml} / \mathrm{Kg} / \mathrm{h}$ for more than $6 \mathrm{~h}$ (less than $12 \mathrm{~h}$ ). Inclusion criteria for early CKD were based on the GFR which ranged from $45 \mathrm{ml} / \mathrm{min} / 1.73 \mathrm{~m}^{2}$ to $59 \mathrm{ml} / \mathrm{min} / 1.73 \mathrm{~m}^{2}$. AKI+CKD patients 
were judged by clinical examination index as described above. Diabetic CKD, non-diabetic CKD with IgAN $(n=50)$ and non-diabetic CKD without IgAN patients were categorised by diabetic symptoms or IgAN from CKD patients. This study was approved by the Human Medical Ethics Committee of Zhejiang Provincial People's Hospital.

\section{Urine collection, urinary protein extraction and digestion}

In brief, void mid-stream morning urine samples (50 ml for kidney disease samples and $150 \mathrm{ml}$ for the control group) were collected in sterile containers and divided into $10 \mathrm{ml} /$ tube, $1 \mathrm{ml}$ protease inhibitor was added, and samples were centrifuged at $2000^{\prime} \mathrm{g}$ for $20 \mathrm{~min}$ at $4^{\circ} \mathrm{C}$. Supernatants from the same illness were removed, combined, and stored at $-80^{\circ} \mathrm{C}$ for subsequent experiments. Urinary proteins were isolated using the trichloroacetic acid (TCA)-acetone precipitation method, and the concentration of total urinary protein was measured using a bicinchroninic acid (BCA) Protein Assay Kit (23225, Thermo Fisher Scientific, MA, US). Urinary proteins were quantified and the quality was assessed using Bradford assays and sodium dodecyl sulphate-polyacrylamide gel electrophoresis (SDS-PAGE). Based on the quantitation results, $100 \mu \mathrm{g}$ urinary protein was diluted 10 -fold using $0.5 \mathrm{M}$ tetraethylammonium bromide (TEAB) containing $0.1 \%$ SDS and digested with trypsin (50:1 protein/trypsin w/w; Promega, Madison, $\mathrm{WI}$ ) at $37^{\circ} \mathrm{C}$ for $3 \mathrm{~h}$. Peptides were cleaned and redissolved in TEAB/water (1:1 v/v).

\section{iTRAQ labelling}

Peptide concentrations were measured using a BCA kit and sample volumes were adjusted to $15 \mu$ total volume containing $25 \mu \mathrm{g}$ peptides using 0.5 M TEAB. Peptides were labelled using 8-plex iTRAQ reagent according to the manufacturer's protocol (4381663, Sigma, Germany). Briefly, peptides were labelled with iTRAQ reagents for $1 \mathrm{~h}$ at room temperature, and iTRAQ-113, 114, 115, 116, 117 and 118 were used to label peptide samples from patients with AKI, AKI+CKD, diabetic CKD, non-diabetic CKD with IgAN and $\mathrm{N}=$ non-diabetic CKD without IgAN, respectively. Labelled samples were mixed as a pool and dried in a vacuum concentrator.

\section{Mass spectrometric analysis}

Mixed peptides were fractionated by strong cation exchange (SCX) chromatography on a 20AD highperformance liquid chromatography (HPLC) system using a polysulphoethyl column (2.1' $100 \mathrm{~mm}, 5$ mm, $200 \AA$; The Nest Group, Southborough, MA, USA). Peptide samples were diluted 10-fold with buffer A consisting of $10 \mathrm{mmol} / \mathrm{I} \mathrm{KH}_{2} \mathrm{PO}_{4}$ in $25 \%$ acetonitrile (can) at pH 3.0, eluted, and loaded onto the column for separation with a gradient of $0-80 \% \mathrm{SCX}$ buffer $\mathrm{B}\left(10 \mathrm{mmol} / \mathrm{K} \mathrm{KH}_{2} \mathrm{PO}_{4}\right.$ at $\mathrm{pH} 3.0$ in $25 \% \mathrm{ACN}$ containing $2 \mathrm{~mol} / \mathrm{KCl}$ ) in SCX buffer A at a flow rate of $200 \mu \mathrm{l} / \mathrm{min}$ for $60 \mathrm{~min}$. After monitoring the 
absorbance at a wavelength of $214 \mathrm{~nm}$ and $280 \mathrm{~nm}, 20$ SCX fractions were collected per min, pooled, and dried in a vacuum concentrator. SCX fractions were dissolved in buffer A consisting of $5 \%$ CAN and $0.1 \%$ formic acid (FA) and further separated using a QSTAR XL system (Applied Biosystems, Thermo Fisher Scientific, MA, US) combined with a 20AD HPLC system (Shimadzu, Kyoto, Japan). Peptides were separated using a Zorbax 300SB-C18 column at a flow rate of $0.3 \mathrm{ml} / \mathrm{min}$ for $70 \mathrm{~min}$ using a gradient of $5-35 \%$ buffer B ( $95 \%$ acetonitrile, $0.1 \% \mathrm{FA})$ in buffer A ( $5 \%$ acetonitrile, $0.1 \% \mathrm{FA})$. Survey scans such as MS/MS spectra were recorded at a resolution of $100 \mathrm{~K}$ with ranges of $\mathrm{m} / \mathrm{z} 400-1800$, and MS scans were obtained with $\mathrm{m} / \mathrm{z}$ 100-2000 for MS/MS analysis. All experiments were repeated twice according to the workflow diagram shown in Figure 1 (Differential abundance of specific proteins in various types of kidney disease).

\section{Data analysis}

MS data were searched against the UniProt human database using Proteome Discoverer software version 1.4 (Thermo Scientific). All peptides were validated with a false discovery rate (FDR) $<0.01 \%$. Parameters for qualitative and quantitative analysis of urinary peptide samples were set according to general preferences. Trypsin was used with one missed cleavage allowed and Carbamidomethyl was employed as a fixed modification. The tolerance of peptides and fragments were set to $\pm 20 \mathrm{ppm}$ and $0.1 \mathrm{Da}$, respectively. The integration window tolerance was set as $20 \mathrm{ppm}$ according to the Most Confident Centroid method. Other parameters such as Scan Event Filters were set as follows: Mass Analyzer = FTMS, MS Order $=$ MS2, Activation Type $=$ HCD. Protein ratios were calculated as the median of only unique peptides for each protein, and normalised by the median protein ratio. A fold-change of $>1.2$ was applied as a cut-off for identifying DEPs from different kidney diseases.

\section{Gene ontology enrichment and pathway analysis}

Based on database searches, DEPs $(>1.2$ fold-change and $p<0.05)$ were assigned biological functions using BLAST2GO annotation, and annotated proteins were categorised based on biological process, cellular component, and molecular function categories. Fisher's exact tests were performed to evaluate enrichment, and Benjamini-Hochberg adjustment was performed to assess multiple data. The KEGG database was employed for annotating pathways in combination with KOBAS software (version 2.0, KOBAS, LONDON, UK). Identification of DEPs related to proliferation, survival, apoptosis, repair, inflammation and migration was based on published literature.

\section{ELISA assay}


Concentrations of urinary proteins SAA1, C5, HGFAC, APOC1 and Reg3A were measured in samples from each of the five types of kidney disease ( $n=30$ per disease) according to the manufacturer's instructions. ELISA kits for SAA1 (DY3019-05), HGFAC (DY5940-05) and Reg3A (DY1514) were purchased from R\&D (Minnesota, US). APOC1and C5 ELISA kits were obtained from Abcam (ab108808) and BD (557965), respectively.

\section{Statistical analysis}

All data are presented as mean \pm standard error of the mean (SEM) and were analysed using SPSS (Chicago, US) and Graphpad prism software (CA, US). Statistical comparisons among groups were performed using one-way analysis of variance (ANOVA) or Student's t-tests, and $p<0.05$ was considered statistically significant.

\section{Results}

\section{Identification of DEPs in urine from kidney disease patients}

In order to the identify DEPs in urine from patients with multiple kidney diseases, the urinary proteomes of patients with $A K I, A K I+C K D$, diabetic CKD, non-diabetic CKD with IgAN, non-diabetic CKD without IgAN, and healthy volunteers were labelled using ITRAQ reagents (Figure 2 Cluster and volcano plot analyses of urinary DEPs identified in the five renal diseases). Cluster analysis of AKI and controls revealed that APOL1, protein S and rheumatoid factor were increased 3-fold, while histone H4, neuroblast differentiation-associated protein AHNAK and calcitonin were own-regulated by more than 4 -fold (Figure $2 \mathrm{~A}$ and Table S1, first image). Among urinary DEPs in AKI+CKD patients, NADH dehydrogenase (ubiquinone) 1 alpha subcomplex subunit 5 and follistatin-related protein 3 were increased 3-fold, while pancreatic alpha-amylase, polymeric immunoglobulin receptor and Golgi integral membrane protein 4 were diminished by more than 4-fold (Figure S1A and Table S2, second image). Numerous urinary DEPs were also identified in diabetic CKD, non-diabetic CKD with IgAN, and non-diabetic CKD without IgAN patients. For example, urinary proteins alpha-adducin and chromogranin-A were markedly upregulated in diabetic CKD patients, as were ephrin type-A receptor 4 and profilin-2 in Non-diabetic CKD with IgAN patients, and alpha-1 globin and apolipoprotein C-I in non-diabetic CKD without IgAN samples. Meanwhile, tubulin-specific chaperone A and pro-epidermal growth factor were significantly downregulated in diabetic CKD patients, as were secreted and transmembrane protein 1 and tumourassociated calcium signal transducer 2 in non-diabetic CKD with IgAN patients, and histone H2B type 1-K and Nectin-4 in non-diabetic CKD without IgAN samples (Figure S2A-S4A and Table S3-S5, images 3-5). Based on analysis of DEPs using volcano plots, 31, 22, 73, 29 and 45 DEPs were significantly increased by over $20 \%$ in the five kidney disease groups, while 125, 134, 213, 158 and 139 DEPs were downregulated by over 20\% (Figure 2B, Figure S1B-S4B). 


\section{Gene ontology annotation of targeted DEPs}

Urinary DEPs identified in different renal diseases were subjected to BLAST2GO annotation. Urinary proteins were classified based on biological process (BP), cellular component (CC) and molecular function (MF) categories according to GO assignment. In the BP category, the majority of DEPs in AKI, AKI+CKD, non-diabetic CKD with IgAN, and non-diabetic CKD without IgAN groups were mainly implicated in cellular process, response to stimulus, metabolic process and multicellular organismal processing. Biological processes were primarily associated with cellular process, single-organismal process, biological regulation and response to stimulus subcategories. At the CC level, the identified DEPs were mainly associated with cell, organelle, membrane and extracellular region subcategories for all five renal diseases. MF analysis indicated that binding, catalytic activity and receptor activity were the primary functions of DEPs in these renal diseases (Figure 3A-E GO annotation of urinary DEPs identified among various renal diseases.). Unlike urinary DEPs in other diseases, electron carrier activity was an exclusive MF category in AKI+CKD patients (Figure 3B). In comparison with the AKI group, DEPs mediating MF did not include chemoattractant activity in AKI+CKD, non-diabetic CKD with IgAN, or non-diabetic CKD without IgAN patients (Figure 3A, B and 3D, E). Additionally, there were unique MF subcategories in diabetic CKD patients, including protein binding and nucleic acid binding transcription factor activities (Figure 3C).

\section{GO term enrichment analysis of DEPs in different renal diseases}

GO term enrichment analysis of DEPs revealed that all DEPs were linked to a variety of biological functions. The top 10 most significant biological functions in each group are shown in Figure 4 (GO enrichment analysis of DEPs identified in different kidney diseases). As indicated in Figure 4A, singleorganism process, protein binding, multicellular organismal process and developmental process were the main biological functions related to urinary DEPs in AKI patients (Figure 4A). Meanwhile, DEPs in AKI+CKD played an important role in developmental processes, system development and biological adhesion (Figure 4B). Among DEPs mediating biological functions, regulation of response to external stimuli, enzyme inhibitor activity, and endopeptidase regulator/inhibitor activity were the most obvious in diabetic CKD patients compared with the control group (Figure 4C). Multicellular organismal process, developmental process, and tissue development are predominant in non-diabetic CKD with IgAN, and single-multicellular organism process, multicellular organismal development, and metal ion binding are the main biological processes in non-diabetic CKD without IgAN patients (Figure 4D, E). These results suggest that the biological functions regulated by DEPs in these renal diseases are diverse and complex.

\section{KEGG pathway analysis of identified DEPs in different kidney diseases}


Further analysis of biological pathways using the KEGG database revealed 6, 13, 12, 10 and 14 signalling pathways that were enriched among the five kidney diseases (Figure 5 Multiple signalling pathways involved in different kidney diseases). KEGG pathways in the AKI group included complement and coagulation cascades, microRNAs in cancer, transcriptional misregulation in cancer, cysteine and methionine metabolism, starch and sucrose metabolism, and apoptosis-multiple species (Figure 5A). As shown in Figure 5B, PI3K-Akt signalling, ECM-receptor interaction, and proteoglycans in cancer were the primary signalling networks mediated by DEPs in AKI+CKD patients, implying that urinary DEPs in AKI and AKI+CKD participated in different pathways, although starch and sucrose metabolism were common to both groups (Figure 5B vs. 5A). In the diabetic CKD group, complement and coagulation cascades, PI3K-Akt signalling, lysosome, and proteoglycans in cancer were the main DEPs-mediated networks, indicating similarities in signal transduction and cross-talk among diabetic CKD, AKI and AKI+CKD patients (Figure 5C). Meanwhile, tuberculosis, proteoglycans in cancer, Rap1 signalling, and starch and sucrose metabolism pathways were significantly enriched in the non-diabetic CKD with IgAN group, while tuberculosis, alcoholism, Staphylococcus aureus infection, and transcriptional misregulation in cancer were observed in non-diabetic CKD without IgAN patients (Figure 5D, E). Thus, bioinformatics analysis of KEGG pathways revealed commonalities as well as process-specific differences among these renal diseases. Importantly, these results imply that some unique signalling pathway could be targeted for the development of therapies for different renal diseases.

\section{Biomarker discovery and verification of unique DEPs in different renal diseases}

To identify specific protein markers in urine, we performed comparative analysis of all DEPs identified from disease patients to detect specific biomarkers in each of the five diseases (Figure 6 Identification and validation of DEPs specific to different renal diseases). Combined with the results of Venn diagrams, we identified 34 and 35 DEPs that are specifically expressed in AKI and AKI+CKD patients. Upon comparison of AKI and AKI+CKD groups, 17 specific DEPs were identified in diabetic CKD and nondiabetic CKD groups, including non-diabetic CKD with or without IgAN patients. In the CKD group, 91 unique and abnormally expressed proteins were identified, while 14 specific protein markers were identified in non-diabetic CKD patients. By comparison, we identified 38 and 47 unique urinary protein biomarkers in non-diabetic CKD with IgAN and non-diabetic CKD without IgAN groups, respectively (Figure $6 \mathrm{~A}$ and Table 1). Further biological function analysis indicated that 22 specific DEPs in AKI were closely related to proliferation, apoptosis, survival, inflammation, repair and migration. For example, SAA1 is associated with proliferation, inflammation and migration, while SHBG is involved in progression of proliferation, apoptosis and survival (Table 2). Using this method, 12 unique DEPs including IGHG1 and PAPPA2 were identified in the AKI+CKD group (Table 3). In contrast to AKI and AKI+CKD groups, 12 urinary DEPs from CKD patients participate in the biological processes mentioned above (Table 4). CKD includes diabetic CKD and non-diabetic CKD patients, and 44 and 6 specific urinary protein markers were identified in these group, respectively (Table 5, 6). Among non-diabetic CKD patients, we identified 17 
specific DEPs in patients with non-diabetic CKD with IgAN, and 27 in those with non-diabetic CKD without IgAN $($ Table 7, 8).

Eventually, we chose five urinary protein markers (Serum amyloid A1, SAA1; Complement component 5, C5; Hepatocyte growth factor activator, HGFAC, Since apolipoprotein C-I, APOC1; and Regenerating isletderived protein 3 alpha, Reg3A) for validation to support our present work. In ELISA assays, an increase in SAA1 was only observed in urine from AKI patients, suggesting that SAA1 could be used as an indicator of AKI disease (Figure 6B). C5 upregulation was observed in all five kidney diseases, implying it is a universal protein marker for all five diseases (Figure 6C). In samples from patients with non-diabetic CKD without IgAN, HGFAC levels were significantly enhanced compared with other groups, which indicates that HGFAC is a potential biomarker for early diagnosis of non-diabetic CKD without IgAN (Figure 6D). Based on the results shown in Figure 6E, we speculated that detection of APOC1, which was upregulated in all renal diseases except diabetic CKD, could be used to distinguish diabetic CKD from other kidney diseases (Figure 6E). Enhancement in Reg3A abundance was observed in both AKI and AKI+CKD groups, which suggests that Reg3A differs significantly between AKI and CKD patients (Figure 6F). Importantly, these results were consistent with our MS data.

\section{Discussion}

The urinary proteome consists of more than 1500 proteins that accurately reflect renal physiology and pathophysiology, as well as kidney dysfunction $(21,22)$. Given that urine collection is non-invasive and facile, urinary proteomics is growing in popularity for kidney disease diagnostics (23). Currently, MSbased proteomic analysis the dominant tool for urinary protein biomarker discovery in kidney disease (24). However, at present there are no completely reliable indicators to specifically discriminate between diverse types of renal disease, due to the complexity of urine and renal pathology. In our present study, we discovered specific DEPs in patients with AKI, AKI+CKD, diabetic CKD, and non-diabetic CKD with or without IgAN (relative to healthy controls). Our results provide new evidence for biomarker discovery and could improve kidney disease diagnosis in clinical management.

iTRAQ labelling combined with 2-D LC-MS/MS is widely considered a powerful method for highthroughput protein identification and relative quantitation in various diseases. ITRAQ-based urinary glycoproteome analysis of DN patients identified $408 \mathrm{~N}$-linked glycoproteins showing significant variation, and alpha-1-antitrypsin (SERPINA1) and ceruloplasmin may serve as indicators to distinguish microalbuminuria and normalbuminuria, which could facilitate the individualised care of patients with DN (25). In comparison with healthy volunteers, 30 proteins including alpha-1-antitrypsin, apolipoprotein A-I and CD44 antigen were significantly up- or downregulated in IgA nephropathy patients (26). However, whether there are common or specific urinary DEPs among the three disease types remains unknown. In the present research, we identified 156, 156, 286, 187 and 184 DEPs that were notably up- or downregulated in patients with AKI, AKI+CKD, diabetic CKD, or non-diabetic CKD with or without IgAN, compared with healthy individuals. Comparative analysis identified 34, 35 and 17 specific DEPs in AKI, $A K I+C K D$ and CKD samples, respectively. Further investigation also identified 91 distinct DEPs in DN, 
compared with 14 unique DEPs in non-diabetic CKD. Numerous specific DEPs were also different between non-diabetic CKD with IgAN and non-diabetic CKD without IgAN. Thus, our results could potentially be applied to improve classification and diagnosis of renal disease.

Urinary proteomics studies commonly employ a long series of statistical filters to identify enrichment of biological functions and other processes during biomarker research. GO term enrichment and KEGG pathway analysis are the most common data analysis methods. Previous studies suggest that cyclic helix B peptide (CHBP)-mediated protection in AKI is implicated in multiple molecular functions such as protein binding activity and oxygen transport, and complicated signal transduction pathways such as oxidative phosphorylation and other diverse signalling and physiological pathways (27). In the progression of $\mathrm{DN}$, the decrease in some urinary proteins is presumably associated with impaired secretory function of other organs, such as a reduced excretion of pancreatic amylase and deoxyribonuclease I (28). The DEPs identified in the IgAN group using iTRAQ appear to be involved in several molecular functions including responses to external stimuli and immune responses, as well as multiple signalling transduction pathways such as cytokine-cytokine receptor interaction and ubiquitin mediated proteolysis (29). For the first time, we performed functional and KEGG pathway analysis of identified DEPs among patients with AKI, AKI+CKD, diabetic CKD, and non-diabetic CKD with or without IgAN. GO analysis results revealed almost identical BP, CC MF categories for all five diseases, although DEPs in DN patients are uniquely involved in the regulation of protein and nucleic acid binding transcription factor activity. Protein annotation and KEGG analysis revealed major differences in $\mathrm{BP}, \mathrm{CC}$, and MF categories of DEPs, but also indicated some common molecular functions and signalling transduction pathways among the five diseases. Our findings could assist a possible strategy to better understand differences between the five kidney diseases by studying the specific molecular functions and signalling pathways involved in each disease.

SAA1, an inflammatory protein mainly produced by hepatocytes, is upregulated by factors that enhance inflammatory cytokines (30). In blood circulation, it is closely related to high-density lipoprotein (HDL) and is of great importance in liver metabolism, responses to infection, and renal cell carcinoma (31-33). HGFAC is a serine protease and key determinant in tissue injury and the progression of renal cell carcinoma $(34,35)$. Detailed analysis and filtering of synchronously altered DEPs identified SAA1 and HGFAC as highly expressed in patients with AKI and non-diabetic CKD without IgAN, implying that detection of urinary SAA1 and HGFAC may help to distinguish these two diseases in a clinical setting. C5, a member of the complement system, can moderate lipid metabolism and reduce fibrosis in DN (36). C5 activation is also observed in patients with lupus nephritis and renal cell carcinoma, which suggests that activated $\mathrm{C} 5$ is widespread in kidney diseases $(37,38)$. Since apoCl, encoded by the apoc 1 gene, is expressed primarily in the liver, its plasma concentration is associated with an increased risk of developing DN, and it acts as a key factor in the pathogenesis of glomerulosclerosis $(39,40)$. Our results further confirmed that urinary C5 is a specific marker of multiple kidney diseases. Nevertheless, urinary apoCl could be used for early diagnosis for various kidney diseases except diabetic CKD. Reg3A is mainly increased during pancreatic inflammation and liver carcinogenesis, and during mammalian urinary tract infection, both Reg3 gamma and HIP/PAP are induced, implying that RegA3 might be implicated in 
inflammation responses during renal disease (41). Based on our findings, we concluded that RegA3 upregulation is associated with AKI and AKI+CKD patients, but not CKD patients, regardless of diabetic CKD or non-diabetic CKD status, and with or without IgAN.

\section{Conclusions}

In summary, we performed urinary proteomics analysis of multiple kidney diseases for the first time, and identified numerous urinary proteins that are common to five different types of disease, as well as several unique protein markers for each disease. Importantly, identified and validate SAA1, HGFAC, C5, APOC1 and RegA3 as common or specific biomarkers for different kidney diseases. This work provides fundamental biological information for multiple kidney diseases, and could accelerate the development of this field in the future.

\section{Abbreviations}

AKI, acute kidney injury; CKD, chronic kidney disease; IgA, immunoglobulin A; IgAN, IgA nephropathy; DEPs, differentially expressed proteins; ESRD, end-stage kidney disease; NGAL, neutrophil gelatinaseassociated lipocalin; KIM-1, kidney injury molecule 1; IL-18, interleukin 18; L-FABP, liver-type fatty acidbinding protein; NDN, non-diabetic nephropathy; eGFR, estimated glomerular filtration rate; 8-OHdG, 8hydroxyl-2'- deoxyguanosine; MCP-1, monocyte chemoattractant protein-1; LC-MS/MS, nano-liquid chromatography-tandem mass spectrometry; MS, mass spectrometry; KEGG, Kyoto Encyclopaedia of Genes and Genomes; TCA, trichloroacetic acid; TEAB, tetraethylammonium bromide; SCX, strong cation exchange; FDR, false discovery rate; CHBP, cyclic helix B peptide; SAA1, Serum amyloid A1; HGFAC, Hepatocyte growth factor activator; C5, Complement component 5; APOC1, Since apolipoprotein C-I; Reg3A, Regenerating islet-derived protein 3 alpha.

\section{Declarations}

\section{Ethics approval and consent to participate}

This study was approved by the Human Medical Ethics Committee of Zhejiang Provincial People's Hospital. We obtained the consent from all participants.

\section{Consent for publication}

Not applicable.

\section{Availability of data and material}

The data used to support the findings of this study are included within the article and the supplementary information files. And the original data of proteomics in this paper are available from the corresponding author upon request. 


\section{Competing interests}

The authors declare that they have no conflict of interest.

\section{Funding}

This work was supported by grants from the Major Projects of Science and Technology Department of Zhejiang Province (Grant Number 2014C03047-2), the Natural Science Foundation of Zhejiang Province (Grant Numbers LY16H050005, LZ17H050001, LY17H050008 and Y18H050024), the Project of Scientific Research Foundation of Chinese Medicine (Grant Number 2017ZA010), and the General Project of the Medical and Health of Zhejiang Province (Grant Numbers 2016KYB007 and 2017KY227).

\section{Authors' contributions}

Juan Jin ${ }^{1,2,3}$, Jianguang Gong ${ }^{1,2,3}$, Li Zhao ${ }^{1,2,3}$, Yiwen $\mathrm{Li}^{1,2,3}$, Qiang He

QH conceived the idea; JJ drafted the manuscript; JJ and JG analyzed and interpreted the patient data and the screening results; LZ designed the figures and YW collected the human samples. All authors read and approved the final manuscript.

\section{Acknowledgements}

We would like to thank the native English speaking scientists of Elixigen Company (Huntington Beach, California) for editing our manuscript.

\section{References}

1. D'Amico G. The commonest glomerulonephritis in the world: IgA nephropathy. Q J Med. 1987;64(245):709-27.

2. Yu SM, Bonventre JV. Acute Kidney Injury and Progression of Diabetic Kidney Disease. Adv Chronic Kidney Dis. 2018;25(2):166-80.

3. Cui Y, Liu S, Cui W, Gao D, Zhou W, Luo P. Identification of potential biomarkers and therapeutic targets for human IgA nephropathy and hypertensive nephropathy by bioinformatics analysis. Molecular medicine reports. 2017;16(3):3087-94.

4. Mehta RL, Kellum JA, Shah SV, Molitoris BA, Ronco C, Warnock DG, et al. Acute Kidney Injury Network: report of an initiative to improve outcomes in acute kidney injury. Crit Care. 2007;11(2).

5. Bellomo R, Kellum JA, Ronco C. Acute kidney injury. Lancet. 2012;380(9843):756-66.

6. Bennett M, Dent CL, Ma Q, Dastrala S, Grenier F, Workman R, et al. Urine NGAL predicts severity of acute kidney injury after cardiac surgery: A prospective study. Clin J Am Soc Nephro. 2008;3(3):66573. 
7. Vaidya VS, V R, Ichimura T, Bobadilla NA, Bonventre JV. Urinary kidney injury molecule-1: a sensitive quantitative biomarker for early detection of kidney tubular injury. Am J Physiol-Renal. 2006;290(2):F517-F29.

8. Parikh CR, Mishra J, Thiessen-Philbrook H, Dursun B, Ma Q, Kelly C, et al. Urinary IL-18 is an early predictive biomarker of acute kidney injury after cardiac surgery. Kidney Int. 2006;70(1):199-203.

9. Portilla D, Dent C, Sugaya T, Nagothu KK, Kundi I, Moore P, et al. Liver fatty acid-binding protein as a biomarker of acute kidney injury after cardiac surgery. Kidney Int. 2008;73(4):465-72.

10. Takahashi S, Nakasatomi M, Takei Y, Ikeuchi H, Sakairi T, Kaneko Y, et al. Identification of Urinary Activin A as a Novel Biomarker Reflecting the Severity of Acute Kidney Injury. Scientific Reports. 2018;8.

11. Filip S, Pontillo C, Peter Schanstra J, Vlahou A, Mischak H, Klein J. Urinary proteomics and molecular determinants of chronic kidney disease: possible link to proteases. Expert Rev Proteomics. 2014;11(5):535-48.

12. Shlipak MG, Mattes MD, Peralta CA. Update on Cystatin C: Incorporation Into Clinical Practice. Am J Kidney Dis. 2013;62(3):595-603.

13. Xu GW, Yao QH, Weng QF, Su BL, Zhang X, Xiong JH. Study of urinary 8-hydroxydeoxyguanosine as a biomarker of oxidative DNA damage in diabetic nephropathy patients. J Pharmaceut Biomed. 2004;36(1):101-4.

14. Cohen-Bucay A, Viswanathan G. Urinary markers of glomerular injury in diabetic nephropathy. Int J Nephrol. 2012;2012:146987.

15. Watanabe $H$, Sanada $H$, Shigetomi $S$, Katoh T, Watanabe T. Urinary excretion of type IV collagen as a specific indicator of the progression of diabetic nephropathy. Nephron. 2000;86(1):27-35.

16. Bolignano D, Lacquaniti A, Coppolino G, Donato V, Fazio MR, Nicocia G, et al. Neutrophil GelatinaseAssociated Lipocalin as an Early Biomarker of Nephropathy in Diabetic Patients. Kidney Blood Press R. 2009;32(2):91-8.

17. Tesch GH. MCP-1/CCL2: a new diagnostic marker and therapeutic target for progressive renal injury in diabetic nephropathy. Am J Physiol Renal Physiol. 2008;294(4):F697-701.

18. Julian BA, Wittke S, Haubitz M, Zurbig P, Schiffer E, McGuire BM, et al. Urinary biomarkers of IgA nephropathy and other IgA-associated renal diseases. World J Urol. 2007;25(5):467-76.

19. Mucha K, Bakun M, Jazwiec R, Dadlez M, Florczak M, Bajor M, et al. Complement components, proteolysis-related, and cell communication-related proteins detected in urine proteomics are associated with IgA nephropathy. Pol Arch Med Wewn. 2014;124(7-8):380-6.

20. Surin B, Sachon E, Rougier JP, Steverlynck C, Garreau C, Lelongt B, et al. LG3 fragment of endorepellin is a possible biomarker of severity in IgA nephropathy. Proteomics. 2013;13(1):142-52.

21. Adachi J, Kumar C, Zhang YL, Olsen JV, Mann M. The human urinary proteome contains more than 1500 proteins, including a large proportion of membrane proteins. Genome Biol. 2006;7(9). 
22. Gopal J, Muthu M, Chun SC, Wu HF. State-of-the-art nanoplatform-integrated MALDI-MS impacting resolutions in urinary proteomics. Proteom Clin Appl. 2015;9(5-6):469-81.

23. Thomas S, Hao L, Ricke WA, Li LJ. Biomarker discovery in mass spectrometry-based urinary proteomics. Proteom Clin Appl. 2016;10(4):358-70.

24. Thongboonkerd V, Malasit P. Renal and urinary proteomics: current applications and challenges. Proteomics. 2005;5(4):1033-42.

25. Guo ZG, Liu XJ, Li ML, Shao C, Tao JL, Sun W, et al. Differential urinary glycoproteome analysis of type 2 diabetic nephropathy using 2D-LC-MS/MS and iTRAQ quantification. J Transl Med. 2015;13.

26. Prikryl P, Vojtova L, Maixnerova D, Vokurka M, Neprasova M, Zima T, et al. Proteomic approach for identification of IgA nephropathy-related biomarkers in urine. Physiol Res. 2017;66(4):621-32.

27. Yang C, Liu JJ, Li L, Hu MY, Long YQ, Liu XH, et al. Proteome Analysis of Renoprotection Mediated by a Novel Cyclic Helix B Peptide in Acute Kidney Injury. Sci Rep-Uk. 2015;5.

28. Lewandowicz A, Bakun M, Kohutnicki R, Fabijanska A, Kistowski M, Imiela J, et al. Changes in urine proteome accompanying diabetic nephropathy progression. Pol Arch Med Wewn. 2015;125(1-2):2738.

29. Ning $X Y$, Yin Z, Li ZX, Xu JY, Wang LN, Shen WJ, et al. Comparative proteomic analysis of urine and laser microdissected glomeruli in IgA nephropathy. Clin Exp Pharmacol P. 2017;44(5):576-85.

30. Jensen LE, Whitehead AS. Regulation of serum amyloid A protein expression during the acute-phase response. Biochem J. 1998;334 (Pt 3):489-503.

31. Gabay C, Kushner I. Mechanisms of disease: Acute-phase proteins and other systemic responses to inflammation. New Engl J Med. 1999;340(6):448-54.

32. Paret C, Schon Z, Szponar A, Kovacs G. Inflammatory Protein Serum Amyloid A1 Marks a Subset of Conventional Renal Cell Carcinomas with Fatal Outcome. Eur Urol. 2010;57(5):859-66.

33. Jang WY, Jeong J, Kim S, Kang MC, Sung YH, Choi M, et al. Serum amyloid A1 levels and amyloid deposition following a high-fat diet challenge in transgenic mice overexpressing hepatic serum amyloid A1. Appl Physiol Nutr Me. 2016;41(6):640-8.

34. Miyazawa K, Shimomura T. Proteolytic Cascade for Activation of Hepatocyte Growth-Factor in Response to Tissue-Injury. Seikagaku. 1995;67(5):381-5.

35. Morris MR, Gentle D, Abdulrahman M, Maina EN, Gupta K, Banks RE, et al. Tumor suppressor activity and epigenetic inactivation of hepatocyte growth factor activator inhibitor type 2/SPINT2 in papillary and clear cell renal cell carcinoma. Cancer Res. 2005;65(11):4598-606.

36. Yiu WH, Li RX, Wong DWL, Wu HJ, Chan KW, Chan LYY, et al. Complement C5a inhibition moderates lipid metabolism and reduces tubulointerstitial fibrosis in diabetic nephropathy. Nephrol Dial Transplant. 2017.

37. Xi W, Liu L, Wang JJ, Xia Y, Bai Q, Xiong Y, et al. Enrichment of C5a-C5aR axis predicts poor postoperative prognosis of patients with clear cell renal cell carcinoma. Oncotarget. 2016;7(49):80925-34. 
38. Song D, Guo WY, Wang FM, Li YZ, Song Y, Yu F, et al. Complement Alternative Pathway's Activation in Patients With Lupus Nephritis. Am J Med Sci. 2017;353(3):247-57.

39. Mooyaart AL, Valk EJ, van Es LA, Bruijn JA, de Heer E, Freedman BI, et al. Genetic associations in diabetic nephropathy: a meta-analysis. Diabetologia. 2011;54(3):544-53.

40. Bus $P$, Pierneef $L$, Bor $R$, Wolterbeek R, van Es $L A$, Rensen PCN, et al. Apolipoprotein C-I plays a role in the pathogenesis of glomerulosclerosis. J Pathol. 2017;241(5):589-99.

41. Spencer JD, Jackson AR, Li B, Ching CB, Vonau M, Easterling RS, et al. Expression and Significance of the HIP/PAP and Reglligamma Antimicrobial Peptides during Mammalian Urinary Tract Infection. Plos One. 2015;10(12):e0144024.

\section{Figures}

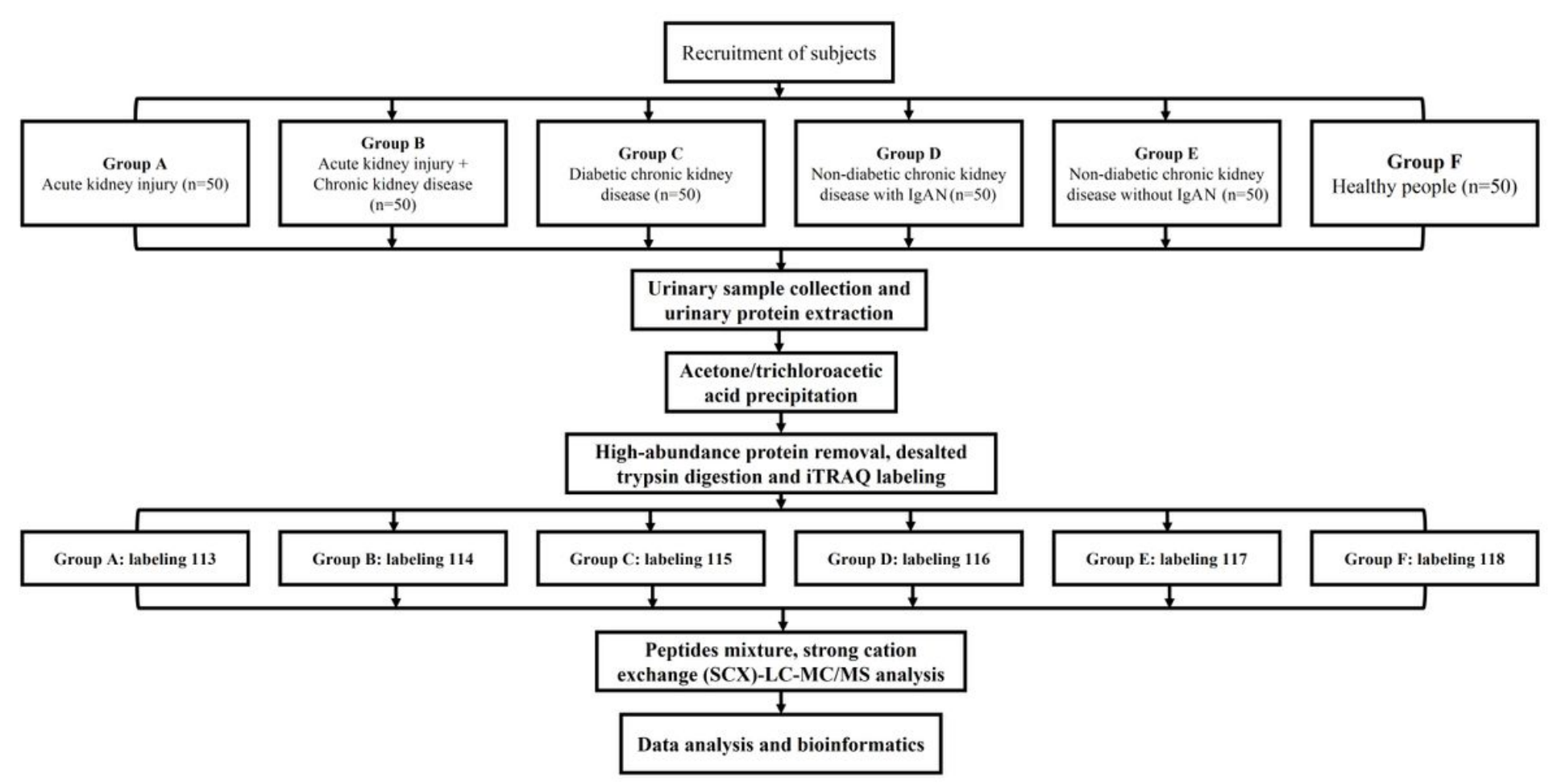

Figure 1

Workflow diagram of the urinary proteomics approach employing Isobaric tags for relative and absolute quantitation (iTRAQ) labelling combined with 2-D LC-MS/MS analysis of samples from patients with AKI, $A K I+C K D$, diabetic CKD, non-diabetic CKD with IgAN, and non-diabetic CKD without IgAN. AKI, acute kidney injury (AKI); $A K I+C K D$, acute kidney injury with chronic kidney disease (AKI+CKD); diabetic CKD, diabetic chronic kidney disease; IgAN, IgA nephropathy. 


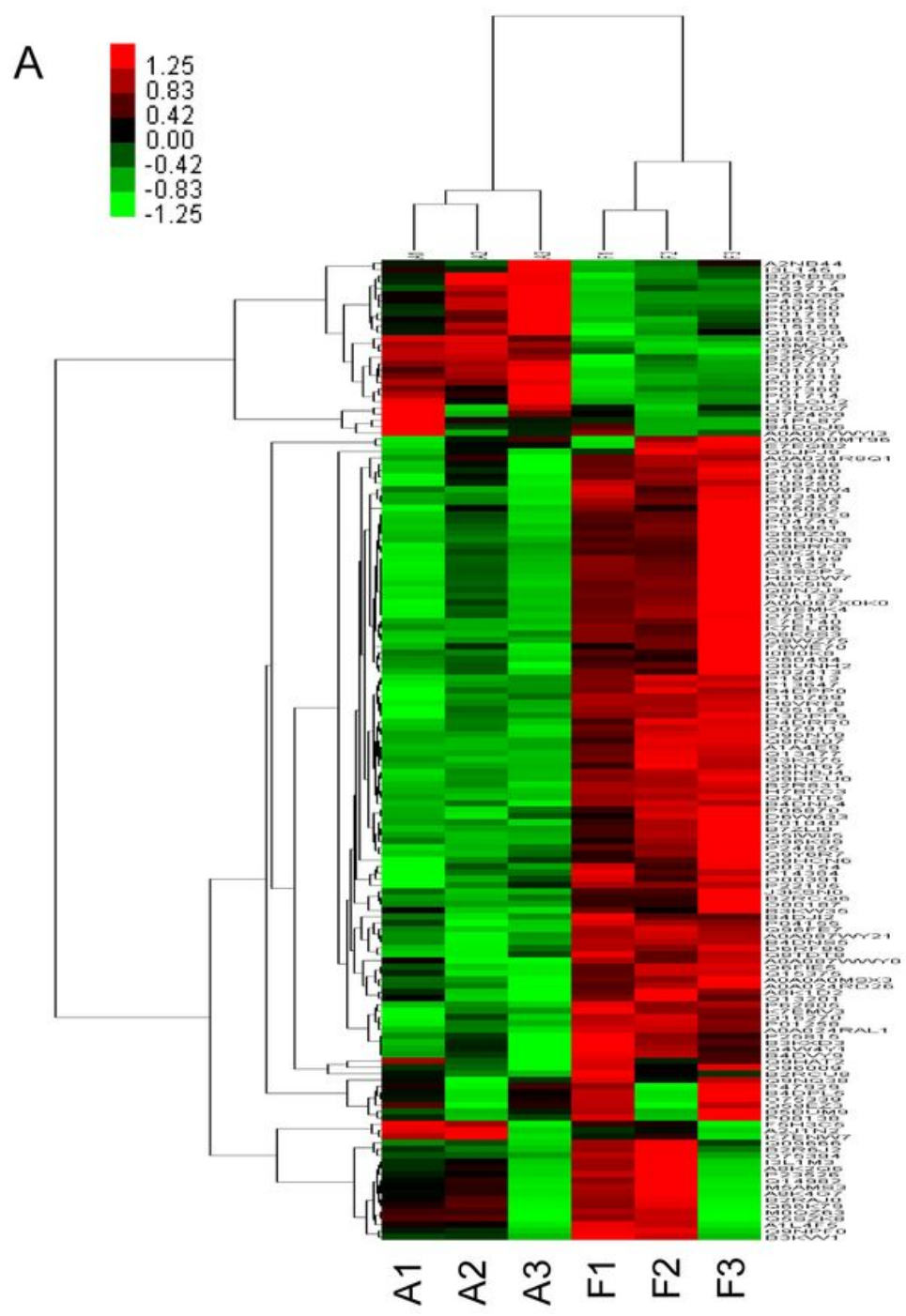

B

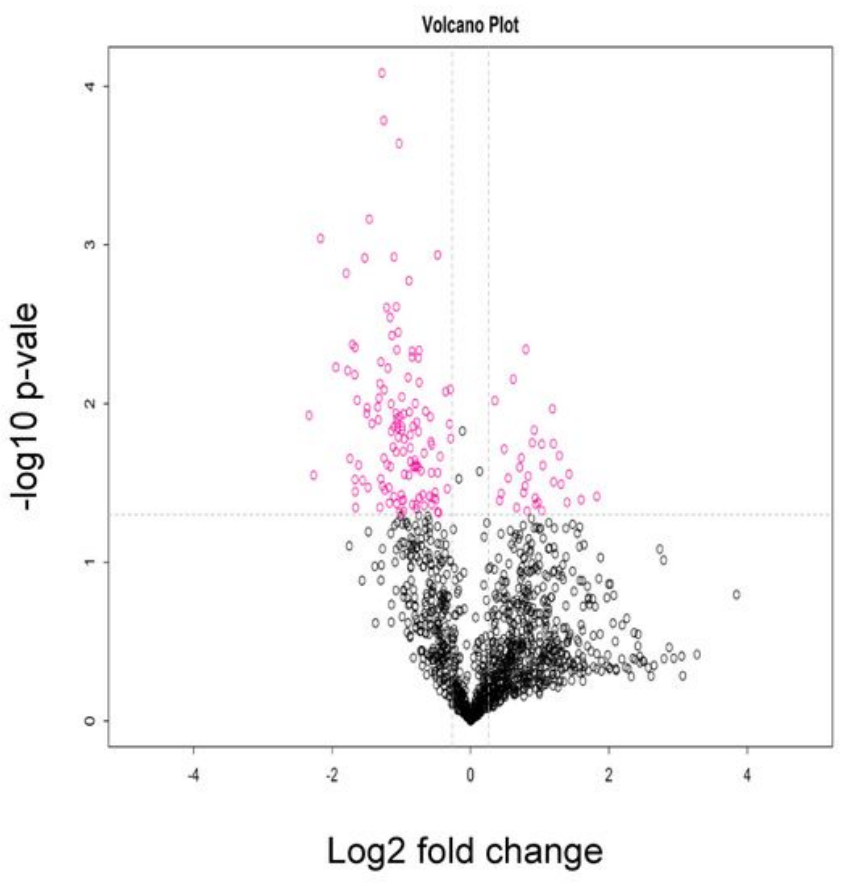

Figure 2

Cluster and volcano plot analyses of urinary Differentially expressed proteins (DEPs) identified in AKI diseases. (A) Cluster analysis of filtered urinary DEPs in AKI diseases compared with healthy volunteers. (B) Volcano plot of all urinary DEPs in AKI and in healthy controls. 
A

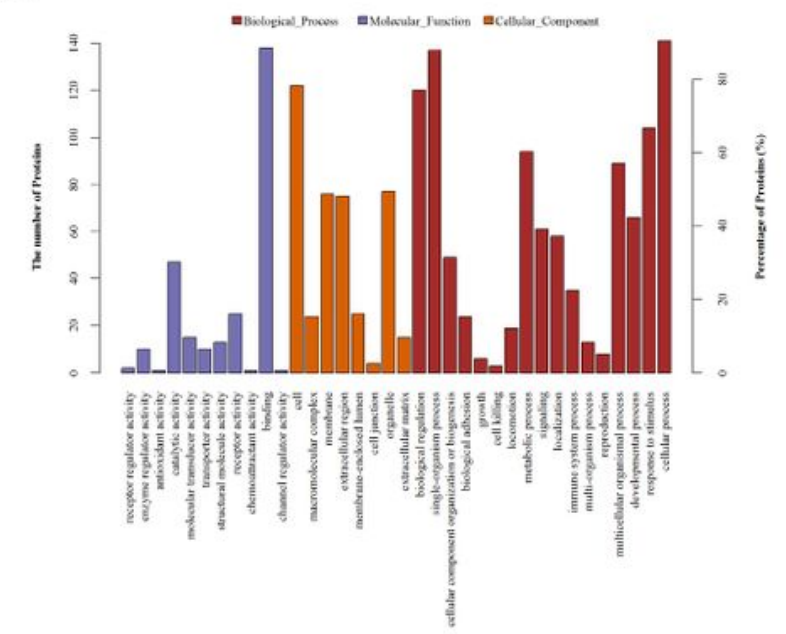

B

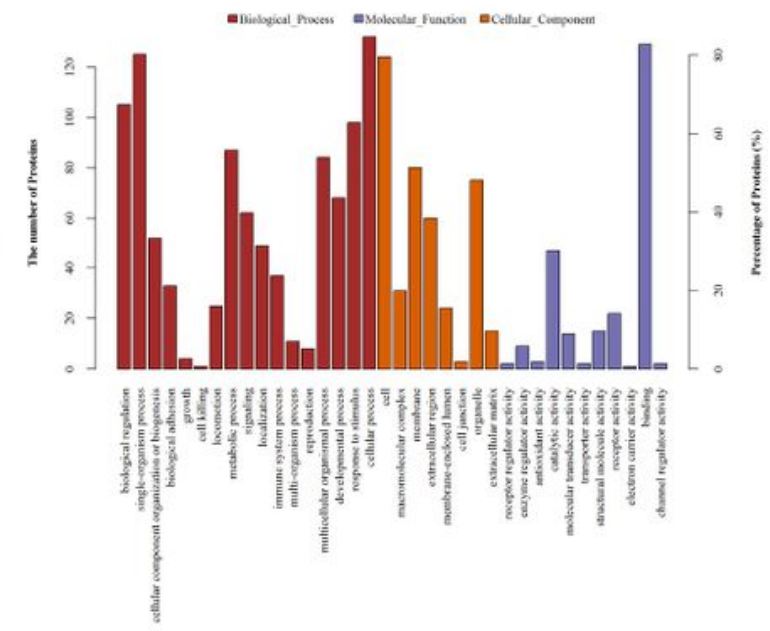

C
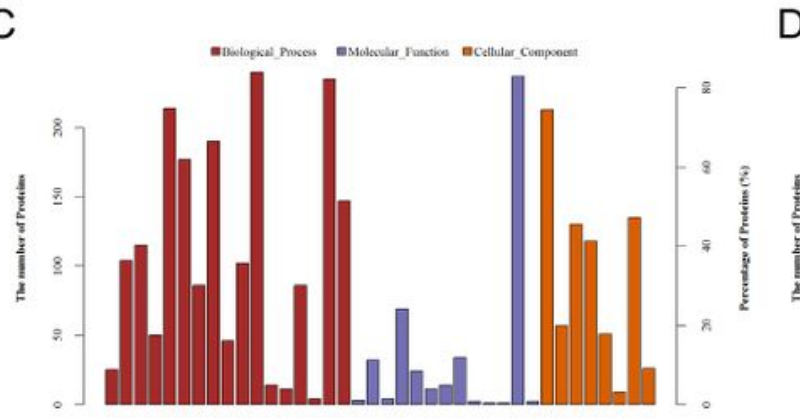

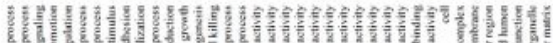

II)

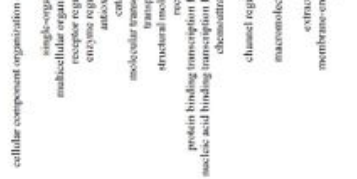

E

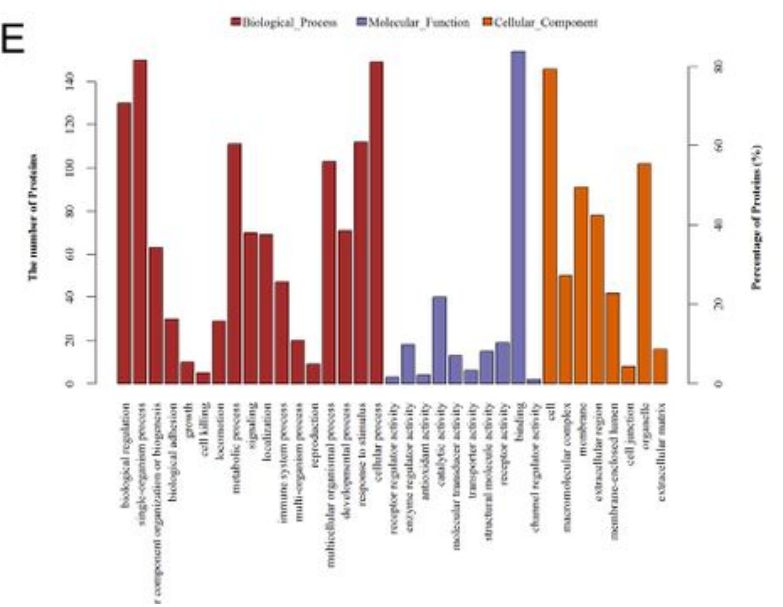

$D$
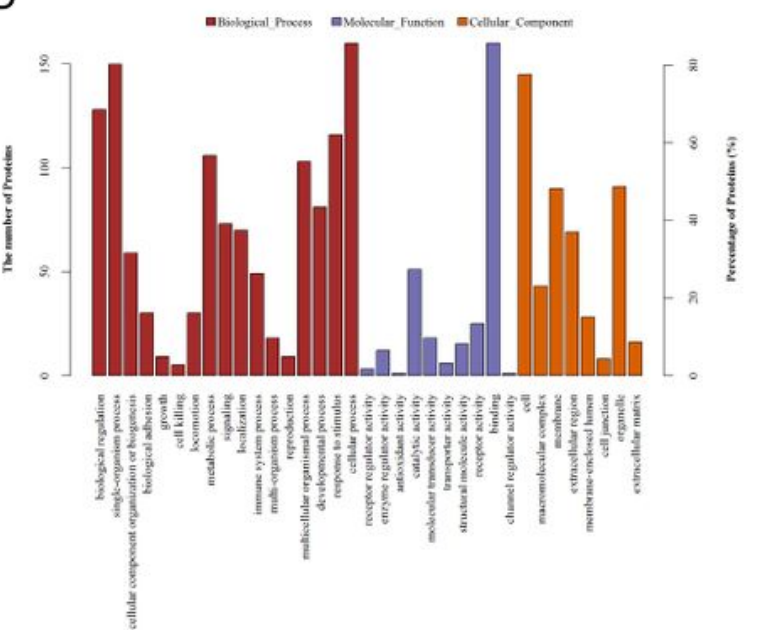

Figure 3

Gene Ontology (GO) annotation of urinary DEPs identified among the five renal diseases. (A) GO annotation of DEPs in AKI compared with healthy controls. (B) GO annotation of DEPs in AKI+CKD compared with healthy controls. (C) GO annotation of DEPs in diabetic CKD in contrast with healthy controls. (D) GO annotation of DEPs in diabetic CKD with IgAN in contrast healthy controls. (E) GO annotation of DEPs in diabetic CKD without IgAN compared with healthy controls. 
A

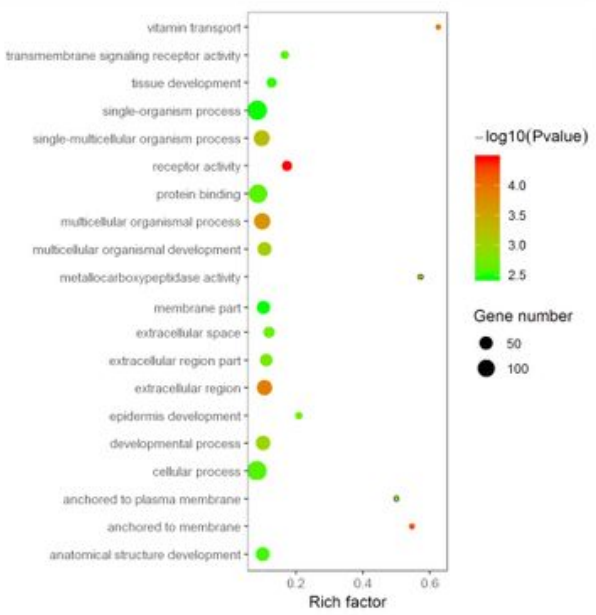

C

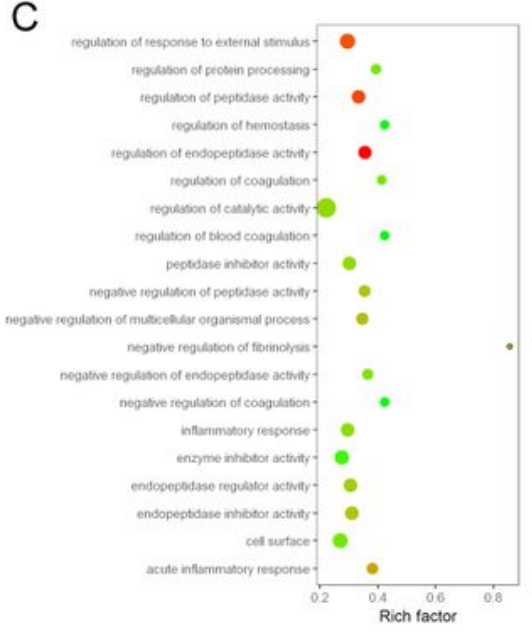

E

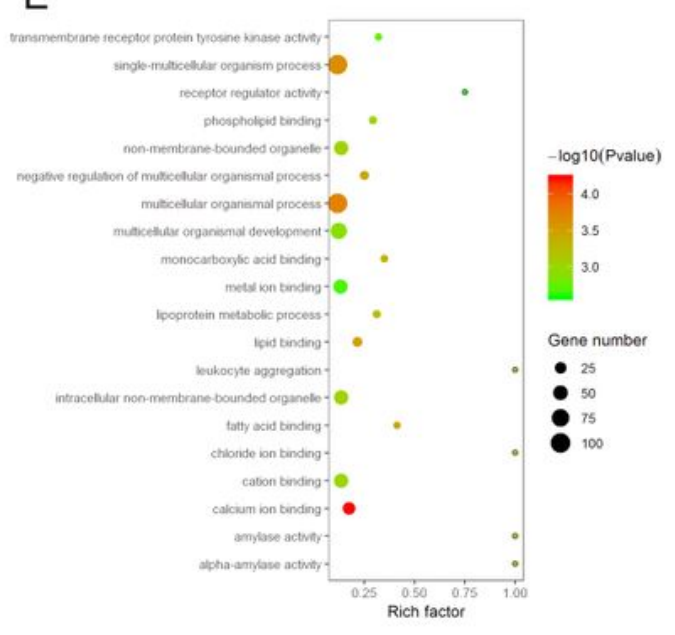

B
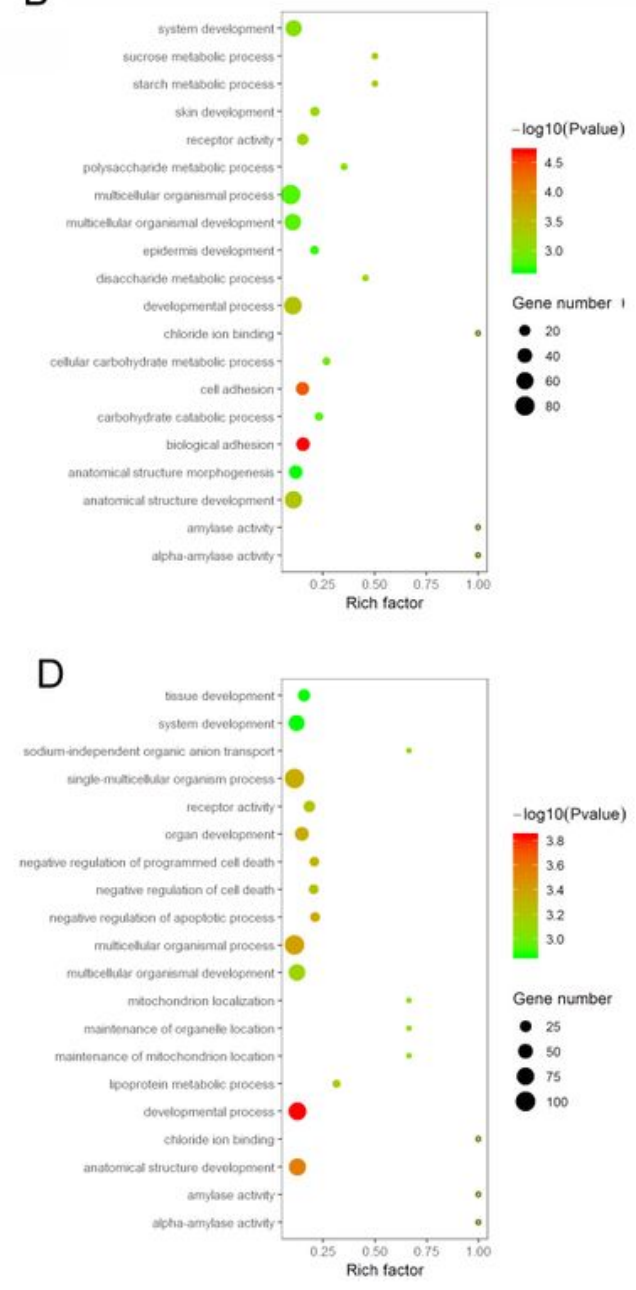

\section{Figure 4}

GO enrichment analysis of DEPs identified in different kidney diseases. (A口E) GO classifications of urinary DEPs in AKI, AKI+CKD, diabetic CKD, diabetic CKD with IgAN, and diabetic CKD without IgAN (Top 10). 
A

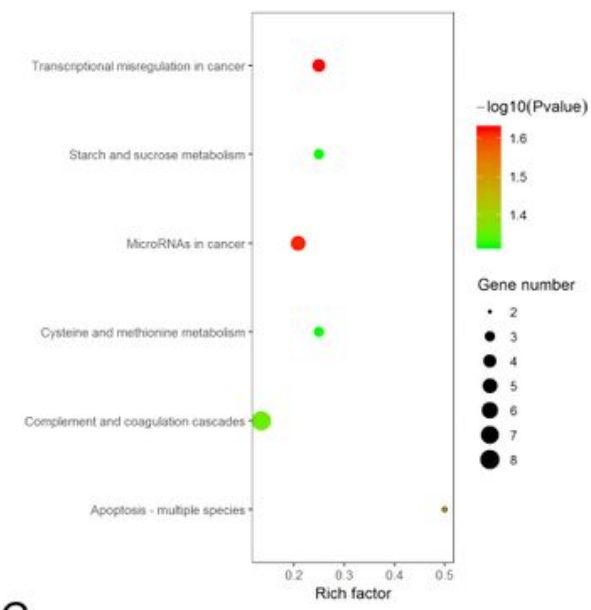

C

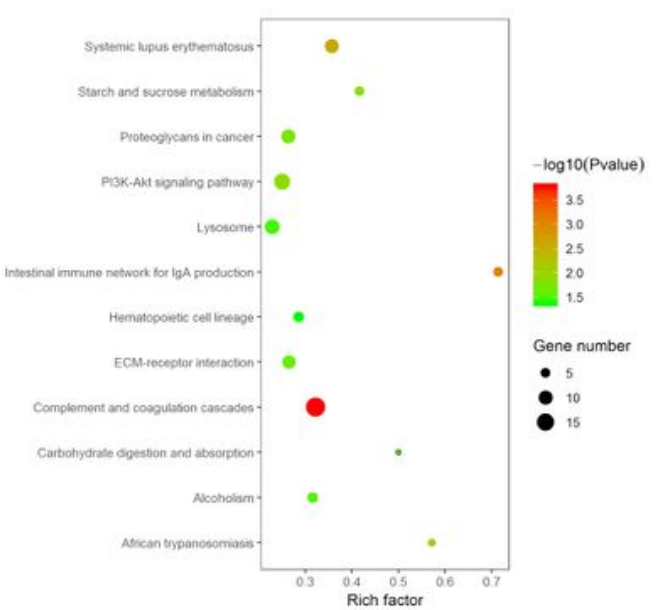

E

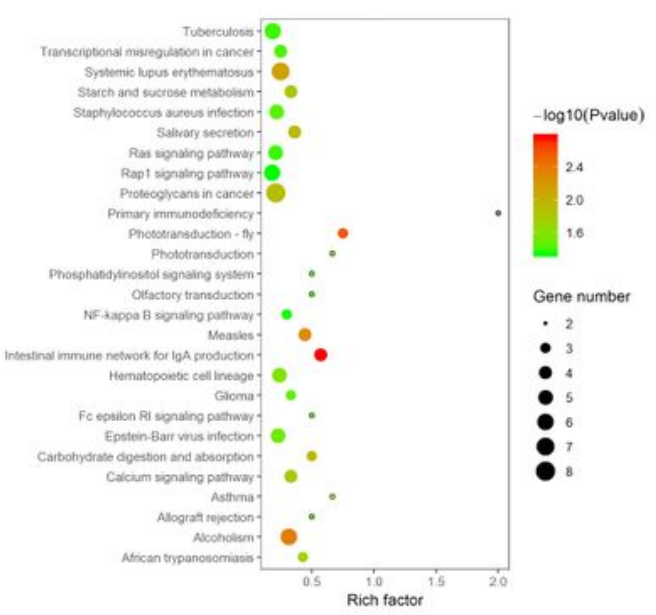

B

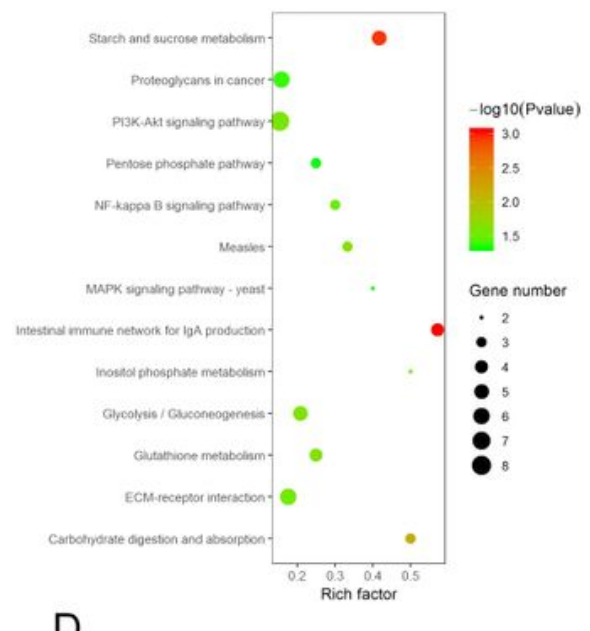

D

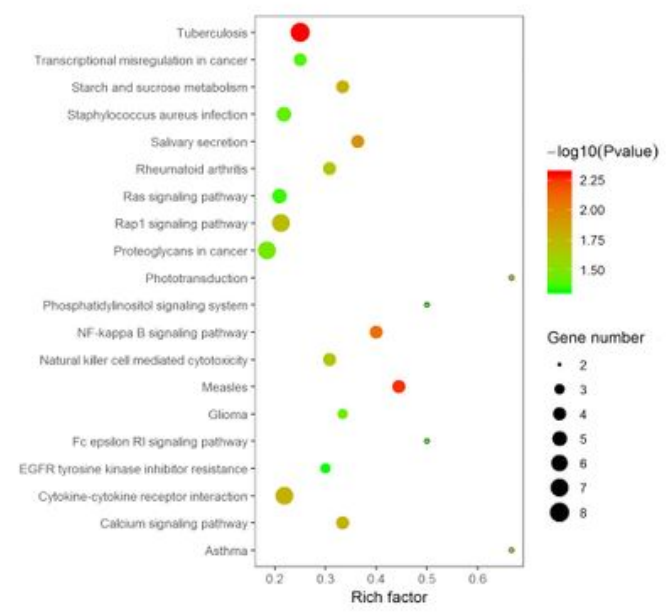

\section{Figure 5}

Multiple signalling pathways involved in different kidney diseases. (A匹E) The main signalling pathways related to urinary DEPs identified in patients with AKI, AKI+CKD, diabetic CKD, diabetic CKD with IgAN, and diabetic CKD without IgAN. 
$\mathbf{A}$

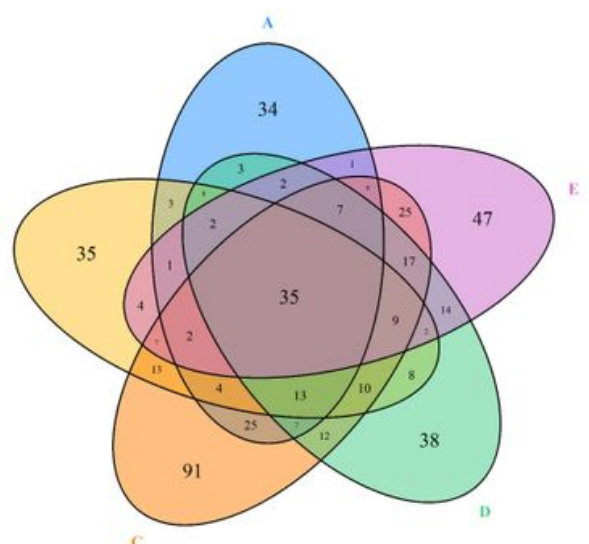

C

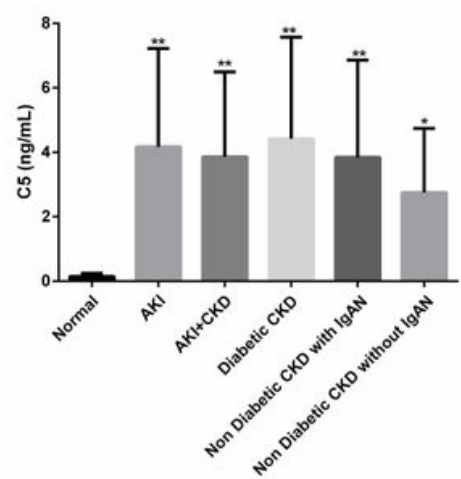

A: AKI vs Ctrl

B: AKI+CKD vs Ctrl

C: Diabetic CKD vs Ctrl

D: Non-Diabetic CKD with IgAN vs Ctrl

E: Non-Diabetic CKD without IgAN vs Ctrl

$\mathbf{E}$
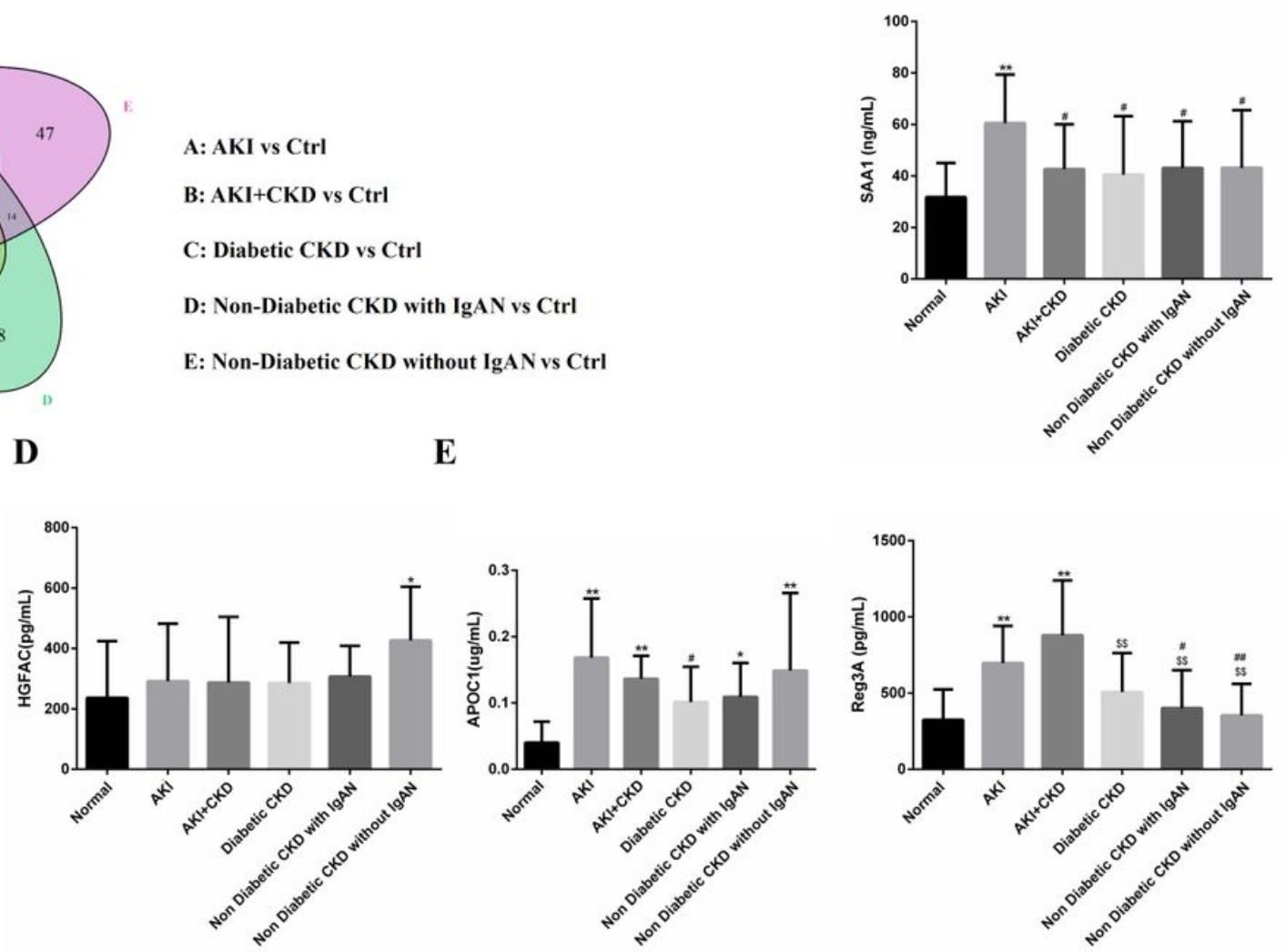

Figure 6

Identification and validation of DEPs specific to different renal diseases. (A) Common and unique DEPs in the five kidney diseases displayed as a Venn diagram. (B口F) Verification of the specific DEPs SAA1, C5, HGFAC, APOC1 and Reg3A using ELISA. $\mathrm{N}={ }^{*} \mathrm{p}<0.05,{ }^{*} \mathrm{p}<0.01$.

\section{Supplementary Files}

This is a list of supplementary files associated with this preprint. Click to download.

- FigureS1S4.docx

- TableS1S13.doc 